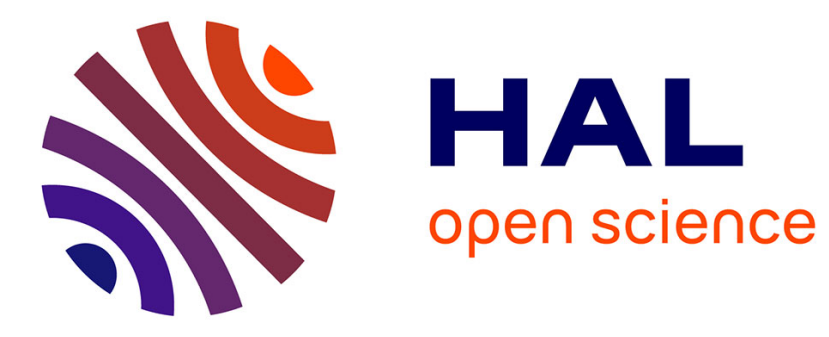

\title{
Macrocycles and Related Hosts as Supramolecular Antidotes
}

\author{
Hang Yin, David Bardelang, Ruibing Wang
}

\section{To cite this version:}

Hang Yin, David Bardelang, Ruibing Wang. Macrocycles and Related Hosts as Supramolecular Antidotes. Trends in chemistry, 2020, 10.1016/j.trechm.2020.08.008 . hal-03054348

\section{HAL Id: hal-03054348 \\ https://hal.science/hal-03054348}

Submitted on 11 Dec 2020

HAL is a multi-disciplinary open access archive for the deposit and dissemination of scientific research documents, whether they are published or not. The documents may come from teaching and research institutions in France or abroad, or from public or private research centers.
L'archive ouverte pluridisciplinaire HAL, est destinée au dépôt et à la diffusion de documents scientifiques de niveau recherche, publiés ou non, émanant des établissements d'enseignement et de recherche français ou étrangers, des laboratoires publics ou privés. 


\title{
Macrocycles and related hosts as supramolecular antidotes
}

\author{
Hang Yin, ${ }^{a}$ David Bardelang ${ }^{\mathrm{b} *}$ and Ruibing Wang ${ }^{\mathrm{a}}$
}

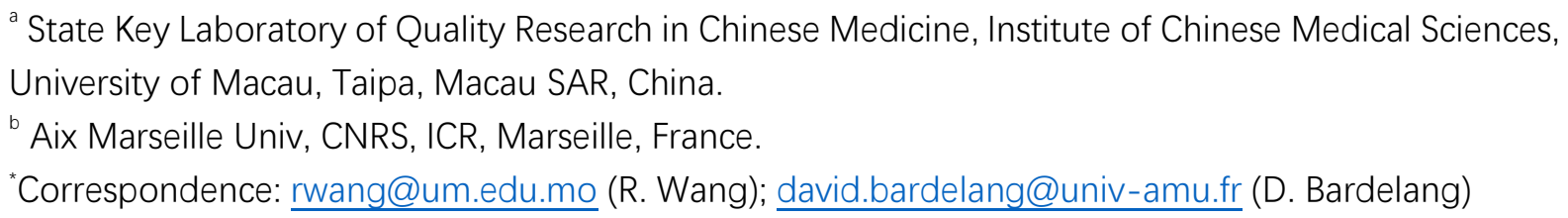

Abstract

Macrocycles and congeners are now being used to reverse the effects of poisonous substances and to alleviate the toxicity of several medicines in vivo. Here we showcase the emergence of this class of compounds behaving as supramolecular antidotes, some of which outperform "gold standards" in the field.

Keywords: Supramolecular Chemistry; Macrocycles; Poisons; Antidotes 


\section{A brief introduction on supramolecular antidotes}

A paradigm shift in the intentional use of poisonous substances for medical applications (e.g., anesthesia) has encouraged the controlled administration of relevant antidotes to cancel the transient "toxic" effects and restore "normal" status. Many poisonous substances are small molecules amenable to be captured by macrocycles via host-guest interactions (Figure 1). In this context, supramolecular antidotes refer to artificial receptors, often macrocycles or congeners, that can specifically trap toxic guest molecules in vivo, thereby effectively reversing or inhibiting their toxicities. Research on synthetic receptors has expanded tremendously since the discovery of the first macrocycles, and several receptors have shown low toxicity along with highly selective affinities for several poisons. Emerging applications along this line of research deal with the use of macrocycles and congeners to (i) alleviate several kinds of medicine's toxicities, eventually decreasing their side effects, and (ii) reduce the impact of suicidal, homicidal, or accidental poisoning (such as pesticide poisoning). Finally, these host compounds have recently been reported to efficiently capture illicit drugs, therefore alleviates alleviate acute toxicity caused by overdose on illicit drugs may also become possible.

\section{The first successful case: a cyclodextrin derivative}

Cyclodextrins (CDs) are cyclic oligosaccharides possessing a hydrophobic cavity capable of complexing a large variety of guest molecules. These compounds are characterized by appropriate aqueous solubility, low toxicity, and facile functionalization due to the presence of numerous primary and secondary hydroxyl groups. In fact, most CDs often show rather modest affinities towards their guest molecules, making them unsuitable for specifically capturing poisonous molecules in complex physiological environments. Sugammadex, a $\gamma$-CD derivative consisting of 8 glucopyranose units each carrying a thiopropionate group, was precisely designed and synthesized to strongly bind rocuronium and vecuronium, two aminosteroid-based neuromuscular blocking agents (NMBAs) (Figure 2) [1]. Rocuronium and vecuronium are extensively used for surgeries to relax skeletal muscles of patients, ensure their immobility, and facilitate endotracheal intubation. This muscle relaxation needs to be terminated after surgeries to limit allergic reactions and prevent the occurrence of various side-effects, some of which can be life-threatening. These NMBAs can be encapsulated by Sugammadex with affinities of $1.79 \times 10^{7}$ and $5.72 \times 10^{6} \mathrm{M}^{-1}$, respectively, enabling this artificial receptor to competitively capture NMBAs from their pharmacological receptor, the nicotinic ACh receptor [1]. Sugammadex was shown to effectively reverse the neuromuscular blocking effects of NMBAs facilitating the recovery from anesthesia [2]. The safety and efficacy of Sugammadex has been demonstrated in clinical studies and it has been successfully commercialized under the brand name Bridion. Sugammadex is now one of the key antidotes to counteract the action of several aminosteroid-based NMBAs in clinical practice. This success paved the way for the development of a growing family of emerging supramolecular antidotes with significant clinical potential.

\section{Another class of artificial receptors: calixarenes}

Calix $[n]$ arenes $(\mathrm{C}[n] \mathrm{As})$ are a family of cyclic phenolic oligomers with historically limited application in biomedical sciences because the parent members of this family have shown poor water solubility and high toxicity. Several functionalized $\mathrm{C}[n]$ As have been developed for potential antidotal applications. 
For instance, negatively-charged $p$-sulfonatocalix $[n]$ arenes (SC $[n] \mathrm{As}, n=4-8)$ are not only water soluble and much less toxic, but also capable of complexing a variety of cationic guest species with relatively high affinities. Paraquat (PQ) is a commonly used cationic pesticide with well-known toxicity that has caused a significant number of casualties globally as a consequence of accidental ingestion or suicide. Currently, there is no specific antidote against the toxicity of PQ. Interestingly, SC[n]As were shown to alleviate $\mathrm{PQ}$ poisoning in mice presumably by forming inclusion complexes with $\mathrm{PQ}$ (binding constant of up to $10^{5} \mathrm{M}^{-1}$ ) (Figure 2) [3].

Recently, SC[4]A was further shown to be able to significantly improve the survival rate of mice poisoned by a lethal dose of succinylcholine (Sch), the most widely used depolarizing NMBA, likely via supramolecular interactions between SC[4]A and the Sch $\left(K_{\mathrm{a}} \sim 10^{4} \mathrm{M}^{-1}\right)$, suggesting that SC[4]A has potential as a supramolecular antidote [4].

More recently, an oligoethylene glycol functionalized, positively-charged guanidinocalixarene (GC[4]AOEG) was prepared in an attempt to neutralize the toxicity of unfractionated heparin (UFH) [5]. UFH is clinically used as an anticoagulant agent, but when administered in sensitive populations or overdosed, UFH can decrease blood pressure and slow heart rate, potentially causing internal bleeding. The use of the only Food and Drug Administration (FDA) approved UFH neutralizer, protamine sulfate, is not without risks. GC[4]AOEG was shown to effectively reverse UFH-induced excessive bleeding of several external and internal injuries in mice, with no adverse effects observed, highlighting its significant potential for clinical translation.

\section{Old macrocycles, new tricks: cucurbiturils}

Cucurbit $[n]$ urils (CB[ $n] \mathrm{s}, n=5-8$ ) are pumpkin-shaped rigid macrocycles containing a hydrophobic cavity lined with two carbonyl portals, accessible to a large spectrum of guest compounds in water and often showing high binding affinities. It has been over a century since the $1^{\text {st }}$ synthesis of $\mathrm{CB}[6]$, and two decades since the other key members of the cucurbiturils (e.g., $\mathrm{CB}[5], \mathrm{CB}[7]$, and $\mathrm{CB}[8]$ ) were discovered from the same synthetic approach. However, it was not until 2015 that $C B[7]$ was found to reverse anesthesia in zebrafish induced by the FDA approved veterinary drug tricaine mesylate via supramolecular capture (Figure 2) [6]. Recently, CB[7] was demonstrated to moderately improve the overall survival rate of mice poisoned by a lethal dose of Sch, a typical depolarizing NMBA frequently used during general anesthesia [4]. Both of these examples illustrate the potential of $\mathrm{CB}[7]$, unfunctionalized, as a supramolecular antidote in anesthesiology.

In another recent study, $\mathrm{CB}[7]$ was further shown to significantly alleviate the toxicity of PQ by decreasing its levels in the plasma and major organs, thereby reducing the mortality rate and related adverse effects of mice poisoned by PQ [7]. Impressively, CB[7] outperformed activated charcoal, the "gold standard" antidote for the treatment of PQ poisoning.

\section{Adaptable congeners: acyclic cucurbiturils}

Rather unexpectedly, acyclic congeners of cucurbiturils have shown quite preserved binding behavior in a number of cases with physiochemical properties compatible with biological applications [8]. For 
instance, congeners named Calabadion 1 (CLBD1) and Calabadion 2 (CLBD2) are C-shape, flexible analogues of cucurbiturils with good water solubility and low toxicity, and have shown tight binding affinity toward a variety of cationic guest species in an adaptable manner, including rocuronium ( $K_{\mathrm{a}}=$ $3.4 \times 10^{9} \mathrm{M}^{-1}$ for CLBD2) [9], leading to an improved performance to reverse rocuronium-induced anesthesia in vivo compared to the clinical "gold standard", Sugammadex.

Very recently, CLBD1 and CLBD2 were found to bind a range of illicit drugs with high affinities. Particularly, CLBD2 can significantly reduce the hyperlocomotive activity of rats induced by methamphetamine (Figure 2) [10]. Hence, acyclic $\mathrm{CB}[n]$ congeners may have promising applications as versatile supramolecular antidotes against both NMBAs and illicit drugs.

\section{A rising star: pillararenes}

Pillar $[n]$ arenes (PA $[n] \mathrm{s})$ belong to a recently developed class of synthetic macrocycles. The structural tunability of both rims has fueled an ever-increasing pool of structures for a plethora of applications. Some of the PA $[n]$ derivatives exhibit good water solubility, low toxicity, and selective binding affinities for guest molecules, fulfilling the fundamental requirements to be considered as supramolecular antidotes. For instance, water soluble carboxylated pillar[6]arene (WPA[6]) was previously shown to reverse the toxicity of $\mathrm{PQ}$ in vitro [11], and was demonstrated more recently to effectively reverse the toxicity of lethal doses of Sch in mice [4], in a more pronounced manner than that of SC[4]A or CB[7]. In the latter example, WPA[6] remarkably decreased a variety of severe side-effects of Sch in mice (Figure 2). These examples showcase the significant potential of water-soluble PA[ $n]$ s as universal antidotes for cationic drugs and poisonous molecules.

\section{Outlook and challenges}

Upon the successful clinical translation of Sugammadex, scientists have started to investigate macrocycles and congeners as potential supramolecular antidotes for a broad spectrum of medicines and poisons. Several compounds have emerged as excellent antidotes showing promising efficacy and safety profiles in vivo, pushing forward the frontiers of the domain. Despite these preliminary scientific and commercial success, several key questions remain. First, for clinical translation, receptors must be available at a relatively large scale as commercial products. Can some of these clinically promising macrocycles and congeners be made on a sufficient commercial scale? Second, the balance between specificity and generality of an antidote must be carefully considered. Some supramolecular antidotes are specific to some poisons (e.g., Sugammadex) at the expense of the broadness of toxicity reversal. Others, such as cucurbit $[n]$ urils and water-soluble pillar[ $n]$ arenes are more general antidotes towards several types of poisonous molecules; however, lower specificity may lead to unexpected toxicity. Third, can supramolecular antidotes complement traditional ones? Combined therapies based upon both supramolecular capture and pharmacological antidote may provide a new venue for improved treatments of poisoning or chemical toxicity. With the rapid expansion of macrocyclic chemistry, there is no doubt that more and more supramolecular antidotes will be designed and developed in the future, not only for outperforming or complementing the current clinical antidotes in both safety and efficacy, but also extending the scope of poisons that can be treated. 


\section{Acknowledgments}

The Science and Technology Development Fund (FDCT), Macau SAR (Grant No.: 020/2015/A1 and 0007/2020/A), University of Macau (Grant No.: MYRG2019-00059-ICMS), CNRS and Aix-Marseille University are gratefully acknowledged for continuous support.

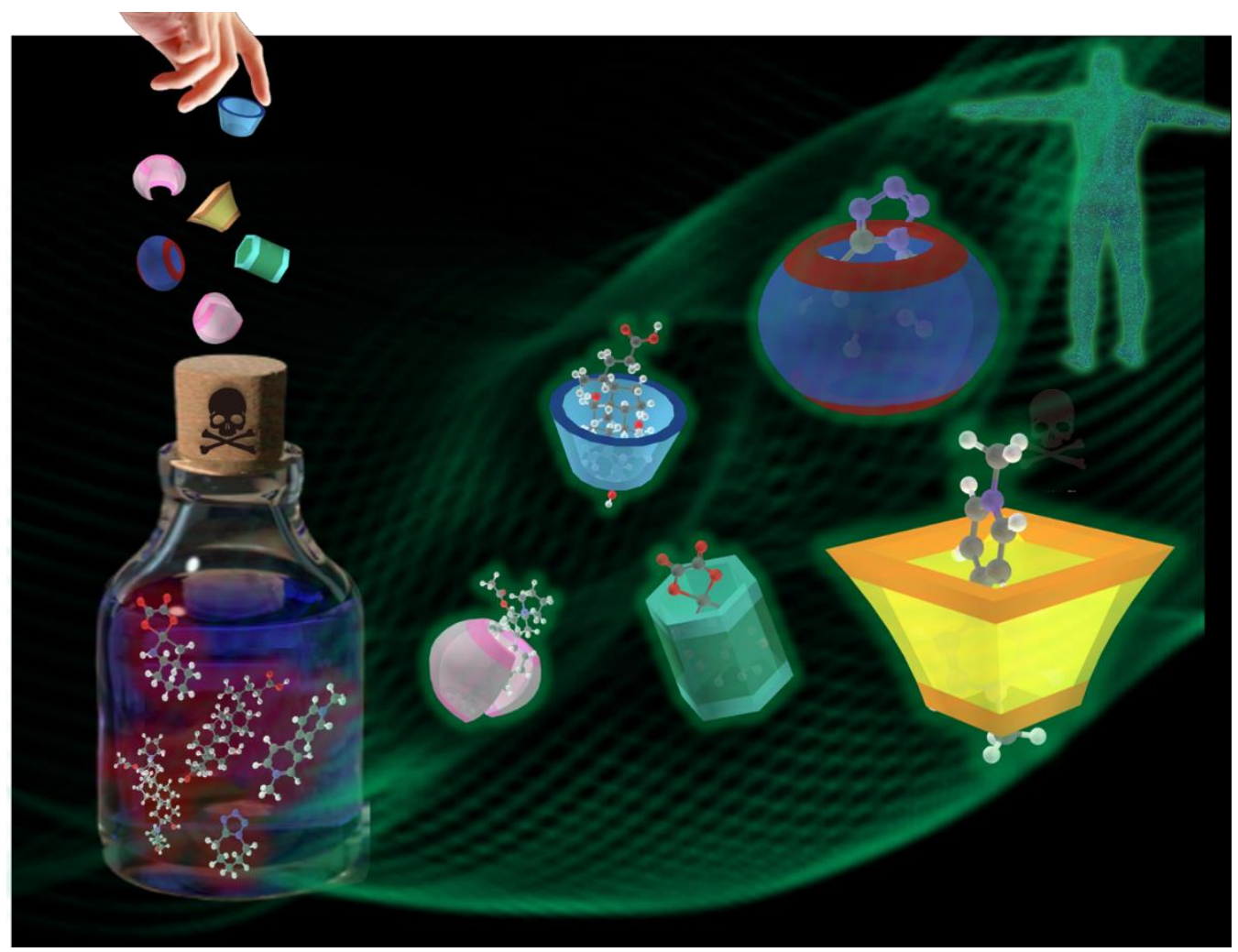

Figure 1. A cartoon of the concept of supramolecular antidotes. The cartoon shows various artificial receptors functioning as supramolecular antidotes to capture poisonous molecules. 


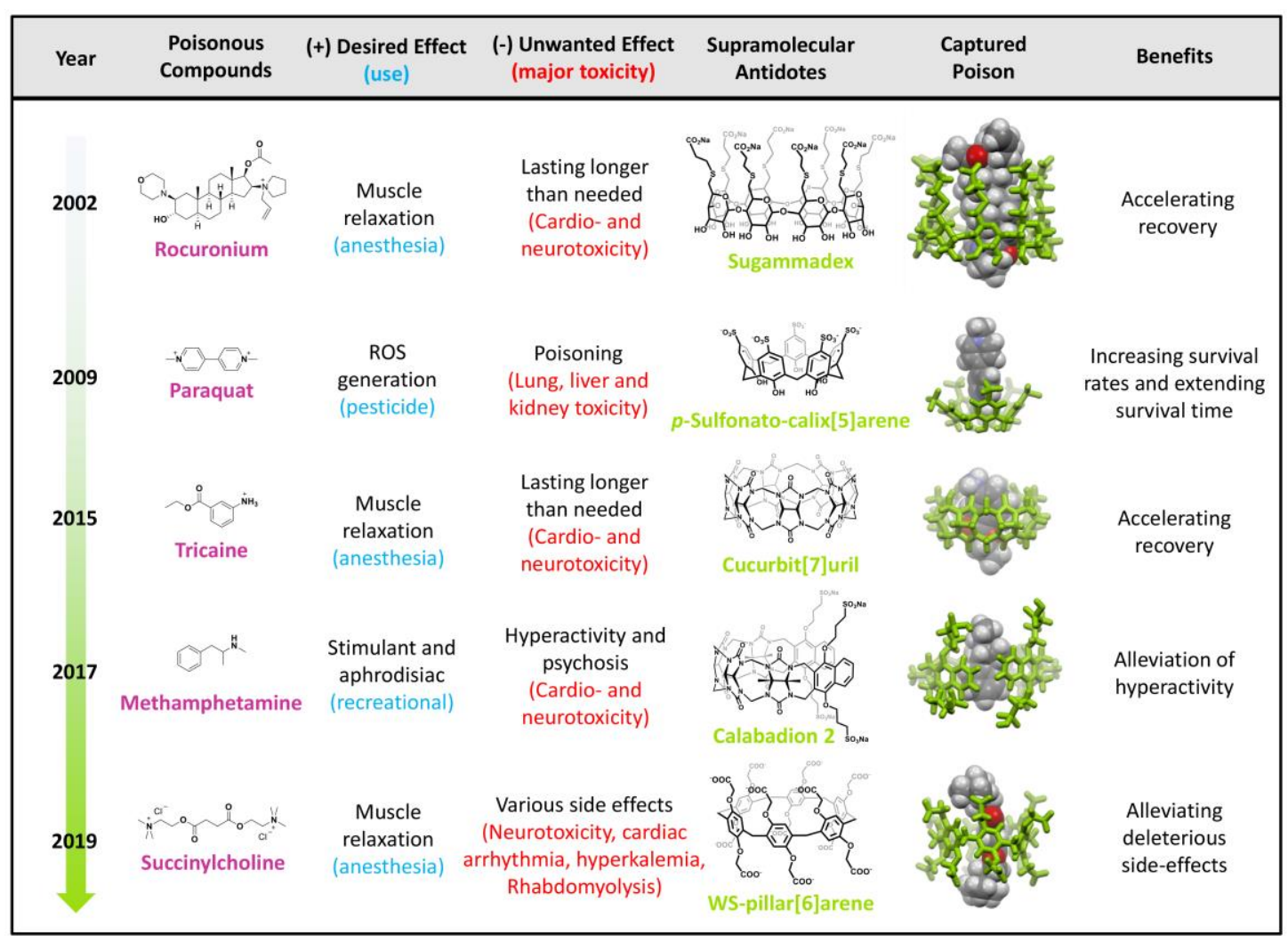

Figure 2. A general timeline of supramolecular antidote development. The timeline demonstrates representative examples from each family of macrocycles and congeners.

\section{References}

1 Bom, A., et al. (2002) A Novel Concept of Reversing Neuromuscular Block: Chemical Encapsulation of Rocuronium Bromide by a Cyclodextrin-Based Synthetic Host. Angew. Chem. Int. Ed., 41, 265270.

2 Adam, J. M., et al. (2002) Cyclodextrin-Derived Host Molecules as Reversal Agents for the Neuromuscular Blocker Rocuronium Bromide: Synthesis and Structure-Activity Relationships. J. Med. Chem., 45, 1806-1816.

3 Wang, K., et al. (2009) Highly Effective Binding of Viologens by $\mathrm{p}$-Sulfonatocalixarenes for the Treatment of Viologen Poisoning. J. Med. Chem., 52, 6402-6412.

4 Zhang, X., et al. (2019) Supramolecular therapeutics to treat the side effects induced by a depolarizing neuromuscular blocking agent. Theranostics, 9, 3107-3121.

5 Huang, Q., et al. (2020) Heparin Reversal by an Oligoethylene Glycol Functionalized Guanidinocalixarene. Chem. Sci., 10.1039/d0sc03922e.

$6 \quad$ Chen, H., et al. (2015) In vivo reversal of general anesthesia by cucurbit[7] uril with zebrafish models. RSC Adv., 5, 63745-63752.

7 Zhang, X., et al. (2019) A Synthetic Receptor as a Specific Antidote for Paraquat Poisoning. Theranostics, 9, 633-645.

8 Ma, D., et al. (2012) Acyclic cucurbit[n]uril molecular containers enhance the solubility and bioactivity of poorly soluble pharmaceuticals. Nat. Chem., 4, 503-510.

9 Haerter, F., et al. (2015) Comparative Effectiveness of Calabadion and Sugammadex to Reverse 
Non-depolarizing Neuromuscular-blocking Agents. Anesthesiology, 123, 1337-1349.

10 Ganapati, S., et al. (2017) Molecular Containers Bind Drugs of Abuse in Vitro and Reverse the Hyperlocomotive Effect of Methamphetamine in Rats. ChemBioChem, 18, 1583-1588.

11 Yu, G., et al. (2012) Pillar[6]arene/Paraquat Molecular Recognition in Water: High Binding Strength, $\mathrm{pH}$-Responsiveness, and Application in Controllable Self-Assembly, Controlled Release, and Treatment of Paraquat Poisoning. J. Am. Chem. Soc., 134, 19489-19497. 